\title{
CCD photometry and long-term optical variability of PKS 2155-304
}

\author{
Y.H. Zhang ${ }^{2}$ and G.Z. Xie ${ }^{1,2}$ \\ 1 CCAST (World Laboratory) P.O. Box 8730, Beijing, 100080, P.R. China \\ 2 Yunnan Observatory, The Chinese Academy of Sciences, P.O. Box 110, Kunming 650011, P.R. China \\ Received April 19; accepted September 25, 1995
}

\begin{abstract}
The paper presents the first evidence for long-term optical variability and colour behaviour of the X-ray-selected BL Lacertae object PKS 2155-304, which is the first example of its class. Our results show that the amplitude of the optical variations of PKS 2155-304 has been only about 1.4 magnitudes from its photometric history; no significant correlation between brightness and colours is found for PKS 2155-304. Our recent CCD photometry of PKS 2155-304 during September-October 1993 and July 1994 showed that it was the same brightness state as that in November 1991 (Smith et al. 1992).
\end{abstract}

Key words: BL Lacertae objects: general individual (PKS 2155-304)

\section{Introduction}

The X-ray-selected BL Lacertae object PKS 2155-304 (=H 2154-304) (Griffiths et al. 1979) is one of the brightest and the most intensively studied objects of its class, and has been one of the most popular extragalactic target for space-based observations at UV and X-ray frequencies (See Treves et al. 1989; Smith et al. 1992, and references therein). It is well known for its short time scales of variability from optical to X-ray wavelengths, and its linear polarization is as high as 10\% (Brindle et al. 1986; Mead et al. 1990; Jannuzi et al. 1993). The spectra of the nebulosity surrounding the PKS 2155-304 yield a redshift $z=$ 0.116 (Falomo et al. 1993).

Miller \& McAlister (1983) found that the optical spectrum softened as PKS 2155-304 brightened. During October and December 1990, the optical continuum was bluer when the object was brighter (Smith \& Sitko 1991), but this trend was not observed by Smith et al. (1992) in November 1991. In September-October 1993 and July 1994, we observed PKS 2155-304 in order to search for short time scales of optical variability. No significant large amplitude variation in timescale of hours was found (Xie et al. 1995).

In all the studies above the results were based on observations taken in a limited time interval and in a limited magnitude range. In this paper we shall present, based on the published $U B V$ data, the long-term variability and colour behaviour of PKS 2155-304 since 1978, when it was identified as a BL Lacertae object. We shall also show if PKS 2155-304 has similar optical variation and colour be-

Send offprint requests to: Y.H. Zhang haviour as observed in OJ 287, which is a classic radioselected BL Lac object (Takalo \& Sillanpää 1989).

\section{Observations and data reduction}

During September-October 1993 and July 1994, PKS 2155-304 was observed with the $102 \mathrm{~cm}$ RCC telescope at Yunnan Observatory equipped with a direct CCD camera. The standard stars in the field of PKS 2155-304 are taken from Smith et al. (1991). The integration times were 2-6 min. The daily-averaged magnitudes and colours are given in Table 1, and all magnitudes observed for PKS 2155-304 each night will be published in another paper altogether with those for other X-ray-selected BL Lacertae objects (Xie et al. 1995). No significant short-timescale and large amplitude variability was detected during our 7 days monitoring.

We have also collected all published ground-based photometric $U B V$ observations and the Fine Error Sensor (FES) $V$ magnitudes on IUE since 1978 (Griffiths et al. 1979; Miller \& McAlister 1983; Brindle et al. 1986; Hamuy \& Maza 1987; Pica et al. 1988; Treves et al. 1989; Mead et al. 1990; Smith \& Sitko 1991; Carini et al. 1992; Smith et al. 1992; Jannuzi et al. 1993; Urry et al. 1993).

\section{Results}

\subsection{Long-term optical variability}

Pica et al. (1988) presented long-term photometry of PKS 2155-304 from July 1979 to November 1986, where the average $B$ magnitude was 13.58 (28 observations), the maximum (brightest) $B$ magnitude 12.93 , and the amplitude 


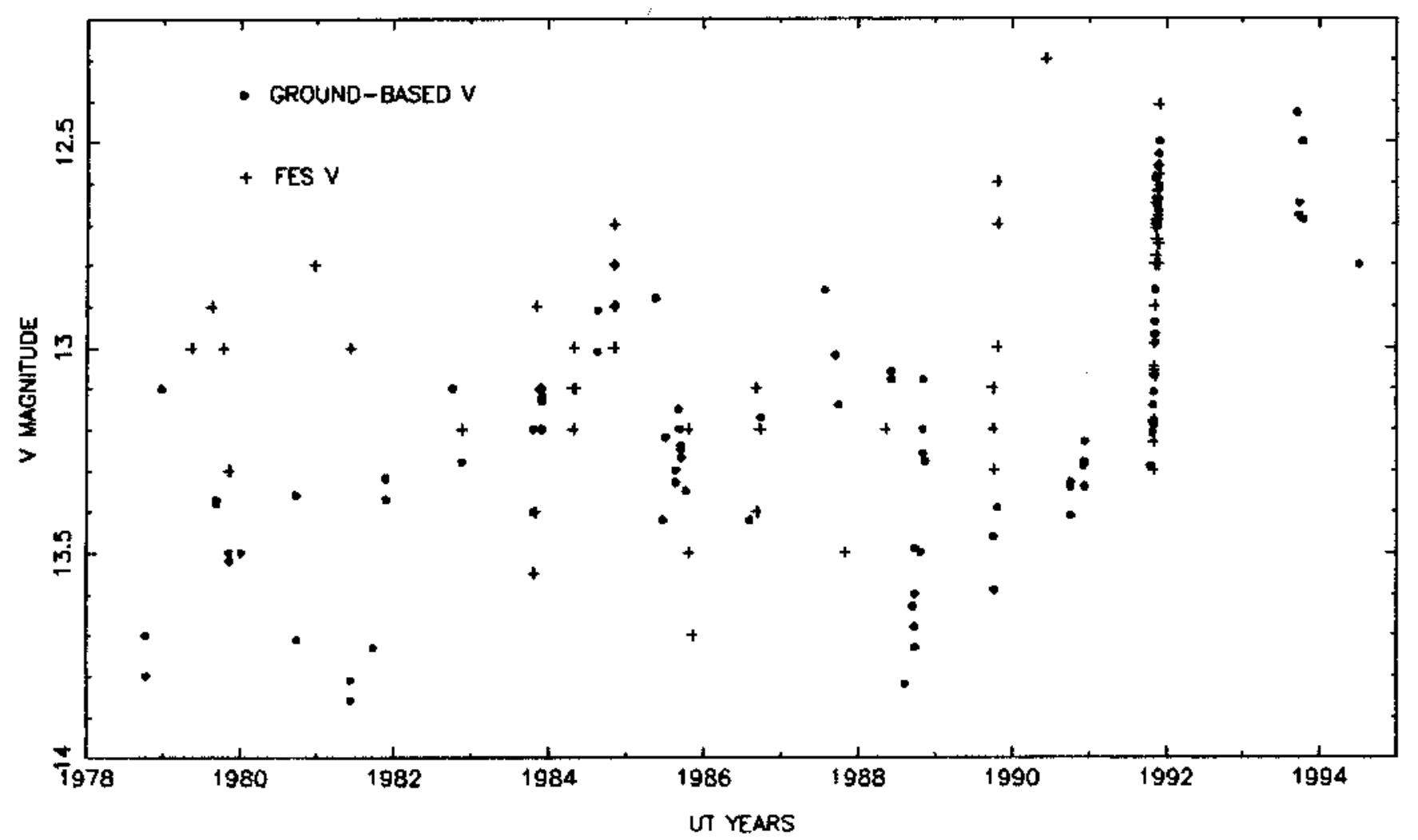

Fig. 1. $V$ magnitude light curve of PKS 2155-304 from 1978 to 1994, collected from the references listed in the text

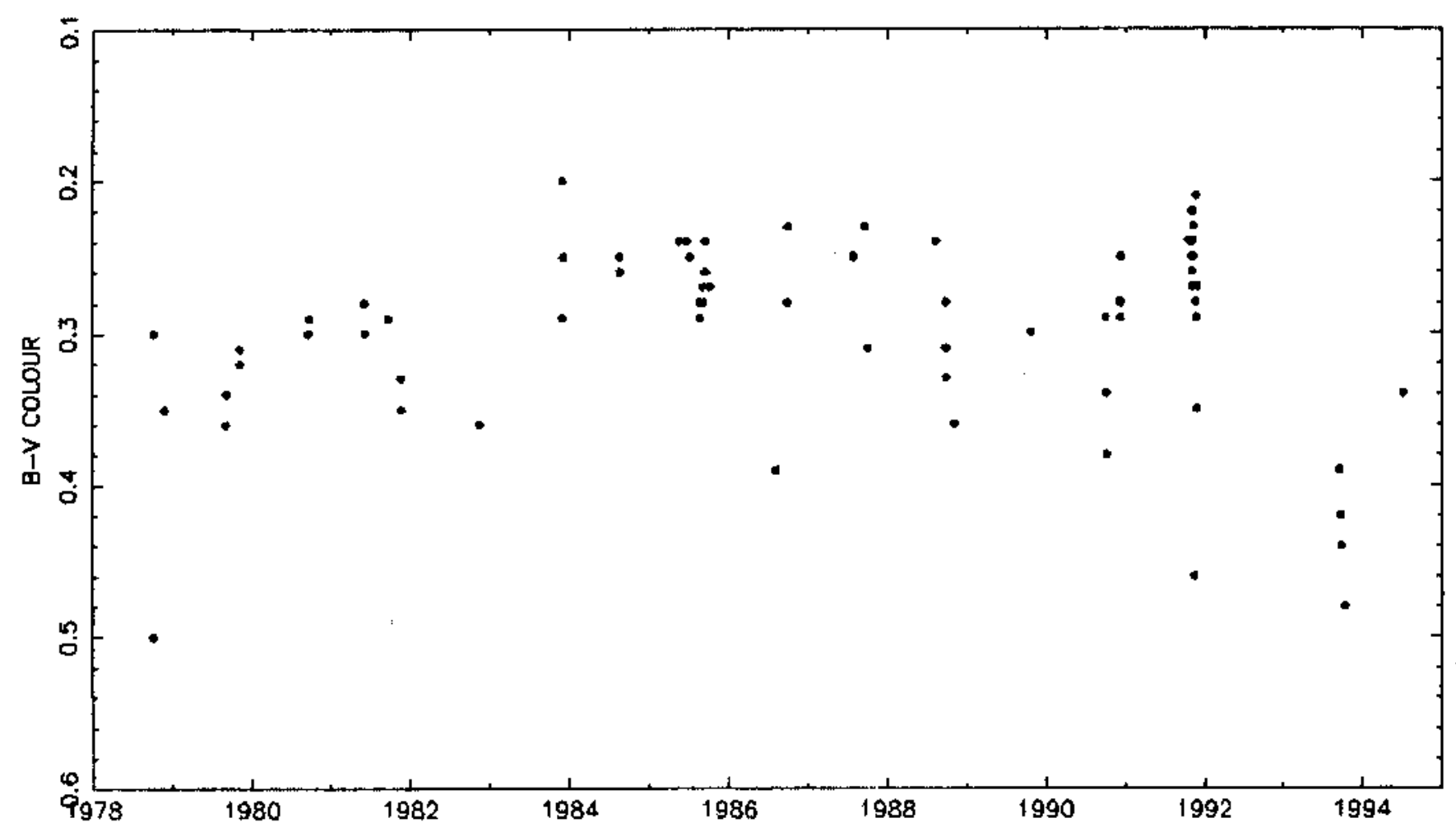

Fig. 2. $B-V$ colour light curve of PKS 2155-304 
Table 1. CCD photometry of PKS 2155-304

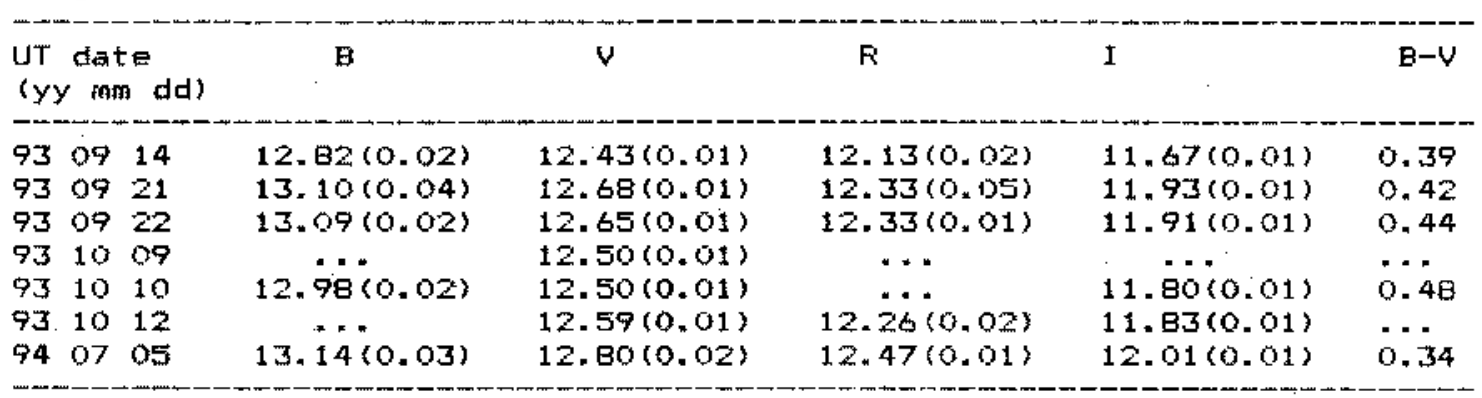

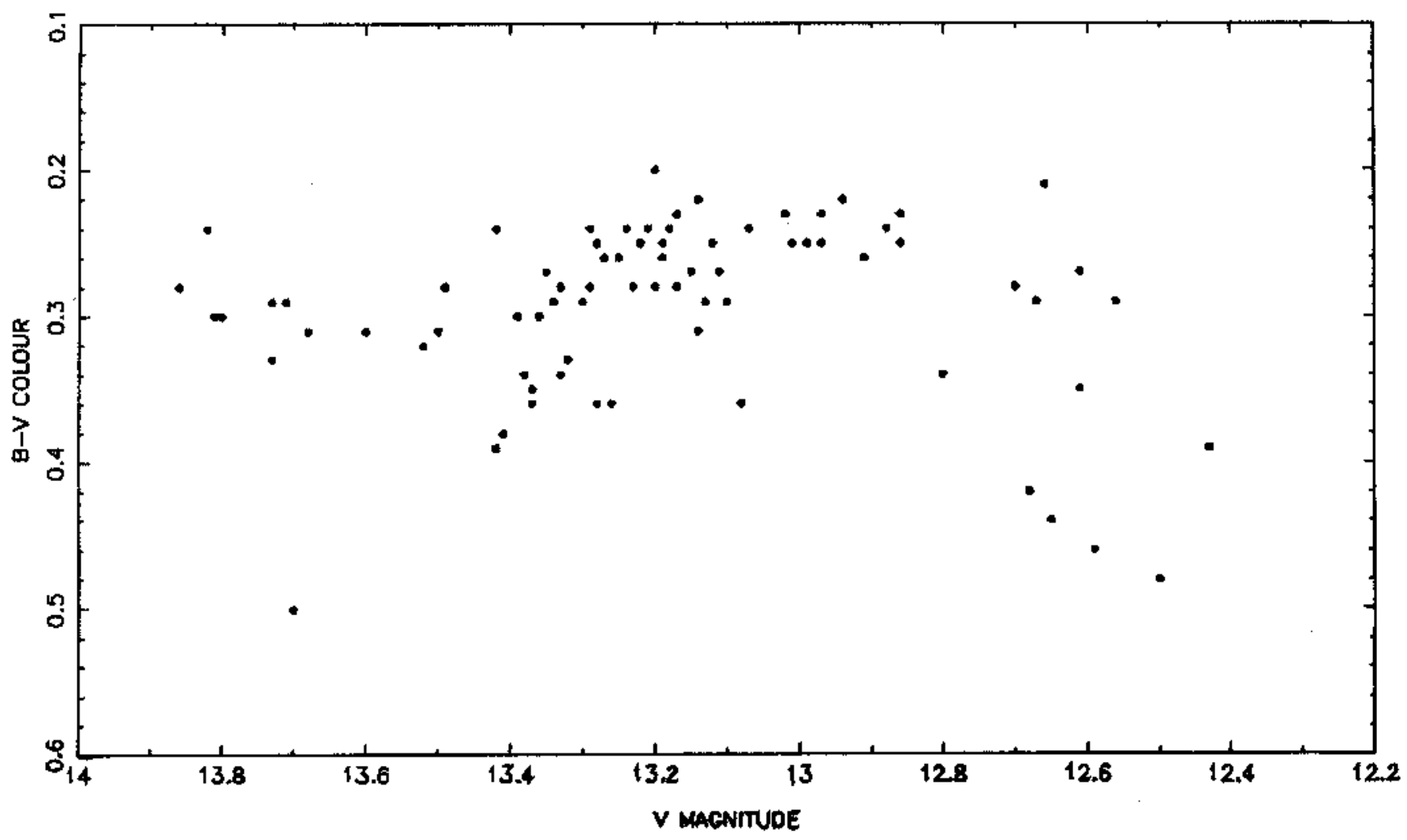

Fig. 3. $B-V$ colour against $V$ magnitude of PKS 2155-304

of variation in $B$ magnitude 1.07. However, neither data nor light curves were published by Pica et al.. So we did not include their data in our analysis of PKS 2155-304.

Light curves based on the other mentioned observations are shown in Fig. 1 for $V$ magnitudes. For groundbased optical photometry, Fig. 1 clearly shows that PKS 2155-304 has a long-term trend of optical variability, that is, PKS 2155-304 brightened from 1978 to 1985, then decreased from 1985 to 1989, and then brightened from 1989; in November 1991 it abruptly brightened (maybe an outburst?), which is the brightest magnitude since it was identified as a BL Lac object. In addition, several smaller variations are also obvious. Our observations in 1993 and
1994 indicated that PKS 2155-304 was still the brightest state since 1978. Also noticeable is that PKS 2155-304 continued to brighten during November 1991 (Smith et al. 1992), which happened to be confirmed by the FES $V$ magnitudes on the IUE satellite during the same period (Urry et al. 1993). We also found good agreement between these two kinds of observations (see Fig. 1). Our observations in 1993 also showed that PKS 2155-304 was still in outburst, but it had decreased about 0.2 mag on 7 July 1994. However, we cannot confirm whether or not the brightest state of PKS 2155-304 in 1993 has a relation to the outburst in November 1991 due to the lack of observations in 1992. The range of variability of PKS 2155-304 
from ground-based observations is $12.82-14.2$ for $B$ magnitudes, and 12.43-13.86 for $V$ magnitudes (data from Griffiths et al. 1979, Carini \& Miller 1992, and Table 1). It shows that the range of the variation of PKS 2155-304 has been only about 1.4 magnitudes during its photometric history, which is much smaller than that of OJ 287 for which the range of the $V$ magnitude is about 12.50-17.00 (Takalo \& Sillanpää 1989).

\section{2. $B-V$ colour indices}

Using the above mentioned observations we have calculated the $B-V$ colour indices for PKS 2155-304. They are displayed in Fig. 2 and Fig. 3. In Fig. $2 B-V$ is shown against time, and in Fig. 3 against $V$ magnitudes. Because we are mainly interested in the overall colour behaviour of PKS 2155-304, we have not included the error bars in the figures. Errors in the averages could be quite large, due to the variability of PKS 2155-304. As can be seen from Fig. 2 the $B-V$ colours of PKS 2155-304 exhibit no obvious changes contrary to OJ 287. The mean range of $B-V$ colours for PKS 2155-304 is $0.24-0.36$.

From Fig. 3, we can see that there are not significant correlations between $B-V$ colour and brightness, which is very different from OJ 287 (see Takalo \& Sillanpää 1989).

However, there are some differences between the observations of Smith et al. (1992) and ours. If we compare the $V-I$ colours of Smith et al. with ours, they are almost identical. But there is a systematic difference of about 0.15 mag if we compare the $B$-values of Smith et al. and ours, whose origin is not clear. Our analysis shows the range of the variation of PKS 2155-304 has been only about $1.4 \mathrm{mag}$, so we cannot expect very large variations in the colours. OJ 287 is a totally different case because in this object the range of the total variation has been almost 6 magnitudes.

\subsection{Comparison with OJ 287}

OJ 287 is a classical radio-selected BL Lacertae object, and is one of the intensively studied objects of its class. Takalo \& Sillanpää (1989) presented the first evidence for long-term optical colour and spectral index variability of OJ 287, and Kidger et al. (1992) reanalyse the 11-year variability period in OJ 287. They found that the $B-V$ colour increased linearly between the years 1972 and 1988 , and that there is a linear dependence of $B-V$ on $V$, which is not found for $U-B$. The range of $V$ magnitude for OJ 287 is about $12.50-17.00$.

PKS 2155-304 shows much smaller daily fluctuations and range in optical brightness than that of OJ 287. The range of the $V$ magnitudes for PKS 2155304 is $12.43-13.86$ in the about 15 years intervals, which is much smaller than that of OJ 287 in the almost same time intervals, the range of the $V$ magnitudes of OJ 287 is $12.50-17.00$ in almost the same time inter- vals as PKS 2155-304. In addition, the $B-V$ colour of PKS 2155-304 ( $B-V: 0.24-0.50)$ is much smaller than that of OJ $287(B-V: 0.30-0.70$; Takalo \& Sillanpää 1989). For the $U-B$ colour , PKS 2155$304(U-B$ : $-0.80--0.60)$ is also smaller than that of OJ $287(U-B$ : $-0.70--0.50$; Takalo \& Sillanpää 1989). Hence, the optical spectrum of PKS 2155304 is bluer than that of OJ 287.

The most dramatic differences between PKS 2155-304 and OJ 287 is found in the fraction of the optical flux that is polarized and the amplitude of the daily variation in polarization. It is rare to find $P>10 \%$ in PKS 2155-304 (e.g. Smith et al. 1992), while OJ 287 spent a large fraction of the time with $P>10 \%$ (e.g. Takalo 1991; Takalo et al. 1992), and the maximum optical polarization observed in OJ 287 is as high as $37.2 \%$ (e.g. Smith et al. 1987). Daily fluctuations in polarization for OJ 287 can be as much as 5 times larger than for PKS 2155-304 (Smith et al. 1992).

\section{Discussion}

We have collected all avaible $U B V$ observations for the X-ray-selected BL Lacertae object PKS 2155-304 to investigate its long-term optical colour behaviour. From these observations we see, unlike the radio-selected BL Lacertae object OJ 287 (Takalo \& Sillanpää 1989), that not only is the total variation very small but also there is no relation between colour and brightness during the photometric history of PKS 2155-304. It would be interesting to understand the reason for these differences between PKS 2155-304 and OJ 287, because they could shed light on the foundamental differences between X-ray-selected and radio-selected BL Lacertae objects.

Using $U B V R I$ photopolarimetric observations (Smith et al. 1992), UV polarimetry (Allen et al. 1993), and the simultaneous optical and ultraviolet variability (Urry et al. 1993), and so on, these authors have completely dismissed the accretion disk models for PKS 2155-304, which were originally proposed by Wandel \& Urry (1991). They have argued that the present observational results are consistent with non-thermal acceleration processes in relativistic plasmas: the relativistic jet is the most likely one for PKS 2155-304, and it can also account for quasi-periodic flaring of about 0.7 days in the ultraviolet flux of PKS 2155-304 (Urry et al. 1993). Therefore, all these observations show that the emission from PKS 2155-304 are dominated by non-thermal radiation, as for OJ 287. However, the comparison of our results for PKS 2155-304 with those for OJ 287 (Takalo \& Sillanpää 1989), shows significant differences, especially in total optical variations and dependence of $B-V$ on $V$, which indicate that PKS 2155304 differs from OJ 287 in some aspects.

Acknowledgements. We are very grateful to Yunnan province foundation of Science and the national foundation of China to support this work. 


\section{References}

Allen R.G., Smith P.S., Angel J.R.P., Miller B.W., Anderson S.F., Margon B., 1993, ApJ 403, 601

Brindle C., Hough J., Bailey J.A., Axon D.J., Hyland A.R., 1986, MNRAS 221, 739

Carini M.T., Miller H.R., 1992, ApJ 385, 146

Falomo R., Pesce J.E., Treves A., 1993, ApJ 411, L63

Griffiths R.E., Tapia S., Briel U., Chaisson L., 1979, ApJ 234, 810

Hamuy M., Maza J., 1987, A\&AS 68, 383

Jannuzi B.T., Smith P.S., Elston R., 1993, ApJS 85, 265

Kidger M., Takalo L., Sillanpää A., 1992, A\&A 264, 32

Mead A.R.G., Ballard K.R., Brand P.W.J.L., Hough J.H., Brindle C., Bailey J.A., 1990, A\&AS 83, 183

Miller H.R., McAlister H.A., 1983, ApJ 272, 26

Pica A.J., Smith A.G., Webb J.R., Leacock R.J., Clements S., Gombola P.P., 1988, AJ 96, 1215
Smith P.S., Hall P.B., Allen R.G., Sitko M.L., 1992, ApJ 400, 115

Smith P.S., Jannuzi B.T., Elston R., 1991, ApJS 77, 67

Smith P.S., Sitko M.L., 1991, ApJ 383, 580

Smith P.S., Balonek T.J., Elston R., Heckert P.A., 1987, ApJS 64,459

Takalo L.O., Sillanpää A., Nilsson K., Kidger M., de Diego J.A., Piirola V., 1992, A\&AS 94, 37

Takalo L.O., 1991, A\&AS 90, 161

Takalo L.O., Sillanpää A., 1989, A\&A 218, 45

Treves A., Morini M., Chiappetti L., Fabrian A., Falomo R., Maccagni D., Maraschi L., Tanzi E.G., Tagliaferry G., 1989, ApJ 341, 733

Urry C.M., et al., 1993, ApJ 411, 614

Wandel A., Urry C.M., 1991, ApJ 367, 78

Xie G.Z., Zhang Y.H., et al., 1995 (submitted to AJ) 